Rev. Elev. Méd. vét. Pays trop., 1971, 24 (3): 381-92

\title{
Étude ultrastructurale des effets produits par diverses drogues trypanocides sur des trypanosomes
}

\author{
par S. M. TOURE $(*)$
}

\begin{abstract}
RESUME
Sont présentés ici les résultats d'observations faites au microscope électronique sur des trypanosomes ayant subi l'action de drogues médicamenteuses in vitro pendant 15 à 18 heures. Trois espèces de trypanosomes ont été soumises à cette expérimentation : Trypanosoma congolense, $T$. brucei et $T$. vivax, et les médicaments utilisés sont : le méthylsulfate de Quinapyramine à raison de $200 \mu \mathrm{g} / \mathrm{mI}$, le Dinamizène $(100 \mu \mathrm{g} / \mathrm{ml})$, le Prothidium $(50 \mu \mathrm{g} / \mathrm{ml})$, l'Ethidium $(25 \mu \mathrm{g} / \mathrm{ml})$, l'Isométamidium $(25 \mu \mathrm{g} / \mathrm{ml})$.

Latteinte des trypanosomes se manifeste par la vésiculisation du noyau et sa fragmentation en mottes. Le kinétoplaste et les structures mitochondriales disparaissent précocement. Le flagelle persiste longtemps, de même que le reticulum endoplasmique et le reticulum sécrétoire. Les ribosomes s'agglomèrent et laissent des plages vides. La membrane cytoplasmique et les microtubules sont peu modifiées.

Ces observations, illustrées à travers 6 planches, sont ensuite discutées et comparées à des travaux portant sur quelques autres espèces de trypanosomes.
\end{abstract}

\section{INTRODUCTION}

Les études menées actuellement sur la virulence des trypanosomes, leur composition antigénique, leur sensibilité à l'égard de médicaments etc. accordent une attention particulière à la morphologie ultrastructurale et la microphysiologie. VICKERMAN, 1965, traite du métabolisme respiratoire de diverses espèces de trypanosomes et trouve des différences de métabolisme en rapport avec la structure des mitochondries et du kinétoplaste. Linfectiosité et la virulence des trypanosomes dépendent en partie de ce métabolisme respiratoire: selon HILL et collab., 1968, les formes virulentes de Trypanosoma brucei, présentes dans le sang, paraissent avoir perdu un cycle de Krebs fonctionnel; leurs mitochondries sont peu développées et les pigments cytochromiques font

(*) Institut d'Elevage et de Médecine vétérinaire des Pays tropicaux, Laboratoire national de I'Elevage et de Recherches vétérinaires, BP 2057, Dakar. défaut; par contre, les formes culturales de ces mêmes espèces ainsi que leurs stades de développement chez l'insecte vecteur présentent un réseau mitochondrial très développé. VICKERMAN, 1965 et 1969 , observe aussi de telles différences. Les organites ultramicroscopiques seraient en outre les supports ou les parties constitutives des antigènes trypanosomiens. BROWN et WILLIAMSON, 1964 trouvent trois antigènes variables dans des fractions mitochondriales de Trypanosoma rhodesiense tandis que VICKERMAN et LUCKINS, 1969, localisent des antigènes variables dans le revêtement superficiel. Ces exemples mettent en relief l'importance des études structurales sur les trypanosomes. Notons en outre que quelques expériences sur le comportement des trypanosomes à l'égard de drogues trypanocides ont été menées sur $T$. rhodesiense (WILLIAMSON et Mc ADAM, 1965; Mc ADAM et WILLIAMSON, 1969). La présente étude porte sur $T$. congolense, $T$. brucei et $T$. vivax. 


\section{MATERIEL ET METHODES}

Trois espèces de trypanosomes ont été utilisées: Trypanosoma (Nannomonas) congolense Broden, 1904; Trypanosoma (Trypanozoon) brucei Plimmer et Bradford, 1899 et Trypanosoma (Dutonella) vivax, Ziemann 1905. Les préparations sont faites à partir de sang de souris ou de rat pour les deux premières espèces et de sang de chèvre pour la troisième,

Les trypanosomes ont été récoltés après adjonction au sang hépariné de sérum de lapin anti-hématies de souris, de rat, ou de chèvre selon le cas, et centrifugation à 3.000 toursminute. Le culot de centrifugation est lavé deux fois avec un tampon phosphate (solution de DU POUEY). Il est remis en suspension dans le tampon phosphate et réparti dans plusieurs séries de tubes à hémolyse. Après centrifugation de ces tubes, le surnageant est jeté pour ne laisser qu'une petite pastille de trypanosomes.

Les dilutions des médicaments trypanocides sont faites parallèlement pour obtenir les concentrations suivantes :

- Methylsulfate

de Quinapyramine (*) . . . $200 \mu \mathrm{g} / \mathrm{ml}$

- Acéturate de Dinamizène (**) $100 \mu \mathrm{g} / \mathrm{ml}$

- Prothidium . . . . . $50 \mu \mathrm{g} / \mathrm{ml}$

- Bromure d'Ethidium . . , $25 \mu \mathrm{g} / \mathrm{ml}$

- Chlorhydrate

d'Isométamidium $\mathrm{Cl} \quad$. . . $25 \mu \mathrm{g} / \mathrm{ml}$

A chacun des tubes renfermant le culot de trypanosomes il a été ajouté $2,5 \mathrm{ml}$ de dilution trypanocide, sauf dans les tubes témoins où le trypanocide a été remplacé par le tampon phosphate.

(*) Antrycide.

(**) Bérénil.

Désignation des tubes de réaction

\begin{tabular}{|l|c|c|c|c|c|}
\hline Espèce & $\begin{array}{c}\text { Quinapyramine } \\
200 \mu \mathrm{g} / \mathrm{ml}\end{array}$ & $\begin{array}{c}\text { Dinamizène } \\
100 \mu \mathrm{g} / \mathrm{ml}\end{array}$ & $\begin{array}{c}\text { Prothidium } \\
50 \mu \mathrm{g} / \mathrm{ml}\end{array}$ & $\begin{array}{c}\text { Ethidium } \\
25 \mu \mathrm{g} / \mathrm{ml}\end{array}$ & Témoin \\
\hline T. congolense & C.Q. & C.D. & C.P. & C.E. & C.T. \\
\hline T. brucei & B.Q. & B.D. & B.P. & B.E. & B.T. \\
\hline T. virax & V.Q. & V.D. & V.P. & V.E. & V.T. \\
\hline
\end{tabular}

Les trypanosomes sont remis en suspension dans chacun des tubes ci-dessus et un contrôle permet de juger de leur vitalité et de leur nombre.

Les préparations sont placées dans l'étuve à $28^{\circ} \mathrm{C}$. La durée des épreuves varie de 15 à 18 heures. Au bout de ce temps, après lecture préalable au microscope photonique, les tubes sont soumis à la centrifugation et les trypanosomes sont traités suivant les techniques de microscopie électronique : fixation par le glutaraldéhyde et par l'acide osmique, inclusion dans l'épon, surcoloration des grilles par l'acétate d'uranyle, observation au microscope O.P.L.

\section{RESULTATS}

Au bout de 15 à 18 heures d'épreuve, les tubes témoins renferment toujours des trypanosomes en grand nombre et de grande vitalité. Ces trypanosomes ont pour la plupart une morphologie en tous points semblable à celle d'éléments fixés sans épreuve médicamenteuse, ni incubation préalable (planche I : Trypanosoma congolense).

Par contre les trypanosomes soumis à l'action des drogues diminuent en nombre et la mobilité des éléments qui survivent est souvent très réduite. L'étude ultrastructurale révèle une 
atteinte marquée dans les organites de leur constitution.

\section{Noyau}

L'enveloppe périnucléaire externe avec ses connexions de réticulum endoplasmique se trouve détachée de la membrane interne et il y a formation d'une poche entre ces deux feuillets. Le contenu nucléaire semble intact dans les cellules où l'atteinte est peu marquée; notamment on distingue la masse nucléaire et le liseré de chromatine périphérique (planche II). Puis l'enveloppe périnucléaire délimite une vésicule dont le volume augmente tandis que le contenu nucléaire se condense (planche III) et se fragmente en mottes de chromatine (planche II C).

\section{Kinétoplaste et mitochondries}

Ces structures semblent subir une atteinte assez précoce car on ne les reconnaît pas nettement dans la plupart des cellules étudiées, alors qu'elles sont apparentes dans les préparations témoins. Il en est de même de l'appareil de Golgi.

\section{Flagelle}

Il reste longtemps apparent quelle que soit sa situation par rapport aux autres organites, et ne présente pas d'altération manifeste.

\section{Organites intracytoplasmiques}

\section{a) Réticulum endoplasmique}

Sur des trypanosomes normaux le réseau endoplasmique est généralement abondant sous forme de :

- Réticulum granulaire émanant de l'enveloppe périnucléaire externe.

- Réticulum agranulaire de sécrétion.

Ce réseau de canalicules subsiste assez longtemps dans la cellule atteinte. Les modifications du réticulum granulaire sont peu marquées et on note encore sa liaison avec l'enveloppe périnucléaire externe (planche IV).

Le réticulum sécrétoire, surtout chez $T$. congolense, est distendu par les produits de lyse des sécrétions élaborées (planche III). A la limite ce réticulum persiste seul, délimitant une enclave vacuolaire. Des figures de myéline dans la cellule altérée (planche IV) semblent dériver de réticulum.

\section{b) Granules divers et ribosomes}

Les granules divers: corps denses bacilliformes ou subsphériques (nomenclature de VICKERMAN), peu abondants à l'état normal, sont par contre nombreux dans un trypanosome altéré. Il y a lieu de discuter de leur nature, car ils pourraient correspondre dans la cellule atteinte à des agglomérats de ribosomes et de corpuscules divers; en effet les ribosomes fusionnent progressivement et forment des amas denses qui laissent tout autour de larges plages agranulaires. Ces plages ne sont pas de même nature que celles qui constituent les réservoirs ou vacuoles, délimités à l'état normal par des membranes qu'on ne voit pas dans le cas des corps denses.

\section{Membrane cytoplasmique}

C'est la structure qui est maintenue le plus longtemps. A l'extrême le trypanosome atteint se présente sous l'aspect d'un sac constitué par la membrane cellulaire renfermant des amas épars de granules et d'éléments réticulaires. Les microtubules pelliculaires sont devenus indistincts. La membrane finit par se rompre et son contenu est libéré ou bien elle reste intacte mais il n'y a plus aucune structure cytoplasmique à l'intérieur.

\section{DISCUSSION}

L'Isométamidium, à la concentration utilisée, a produit des effets plus rapidement que les autres trypanocides, et les grilles qui correspondent à cette série (C.I., B.I., V.I.) sont pratiquement illisibles pour la durée de réaction considérée. Nous n'avons pas tenu compte de cette série.

L'acéturate de Dinamizène, le Prothidium et l'Ethidium ont produit des effets similaires qu'on peut résumer à travers les points suivants :

a) Le décollement de l'enveloppe nucléaire externe qui se trouve séparé du feuillet périnucléaire par un espace de plus en plus grand à mesure que l'atteinte progresse. Ce phénomène pourrait être désigné "vésiculisation du noyau ». Cependant on ne saurait dire que le contenu vésiculaire émane du noyau car si la vésiculisation semble précoce, le nucléoplasme garde assez longtemps sa texture. La dilatation de pores de membrane nucléaire ou une 
solution de continuité à travers cette membrane suggère une origine cytoplasmique du contenu vésiculaire, sans doute introduit dans le noyau par pynocytose du fait d'un défaut de régulation osmotique. Après cette atteinte, le nucléoplasme perd sa texture et se fragmente en mottes. MAC ADAM et WILLIAMSON, 1967, utilisant l'Acriflavine, la Cordycépine et la Pentamidine, observent aussi la fragmentation du noyau de trypanosomes et la dislocation de la chromatine;

b) Le regroupement des ribosomes en amas de densité croissante, ce qui crée une discontinuité dans le cytoplasme où apparaissent des plages optiquement vides. Ces ribosomes finissent par disparaître. ORMEROD, 1962, constate l'agglomération des ribosomes et MAC ADAM et WILLIAMSON, 1967, leur absence en fin de réaction. LANTZ et al, 1968, dans une étude de l'action des trypanocides sur le RNA montrent une interaction des drogues et du RNA soluble, ce qui conduit à l'inhibition de la synthèse des protéines;

c) La disparition des structures mitochondriales (kinétoplaste et mitochondries). Celle, en particulier, du kinetoplaste semble précoce car aucune structure assimilable à cet organite n'a été observée. Plusieurs auteurs ont signalé cette particularité avec la plupart des trypanocides : HERMAN, 1968 : diminution ou disparition du kinétoplaste de Leishmania donovani en culture cellulaire en présence d'acriflavine; KILLICK - KENDRICK 1964: utilisant le Bérénil sur T. evansi; Mc ADAM et
WILLIAMSON, 1967-1969; MUHLPFORDT, 1959 : utilisant la tripaflavine; RIOU, 1968 : disparition de l'ADN du kinétoplaste de $T$. cruzi cultivé en présence de Bromure d'Ethidium. L'atteinte de ces structures mitochondriales, observée dans des conditions expérimentales variées et avec des trypanocides différents, nous semble pour cette raison un paramètre important dans l'étude de la sensibilité des trypanosomes à l'égard des drogues ou du mécanisme d'acquisition de leur résistance aux médicaments;

d) La conservation plus ou moins longtemps du flagelle et des structures membranaires, parmi lesquelles le réticulum endoplasmique et le réticulum sécrétoire.

La Quinapyramine produit la plupart des effets ci-dessus, mais plus lentement. De plus l'atteinte du noyau est peu marquée. D'une façon générale les figures obtenues en $18 \mathrm{~h}$. avec ce médicament montrent moins d'altérations qu'avec les autres.

\section{Remerciements}

Nous tenons à remercier le Laboratoire de Microscopie électronique de la Faculté des Sciences de Dakar, dont le concours a permis la réalisation de ce travail, en particulier feu Monsieur le Professeur BOISSON et Monsieur $\mathrm{X}$. MATTEI, pour leur enseignement et leurs précieux conseils, ainsi que MM. MANFREDI et CHAUVE.

\section{LEGENDE DES PLANCHES}
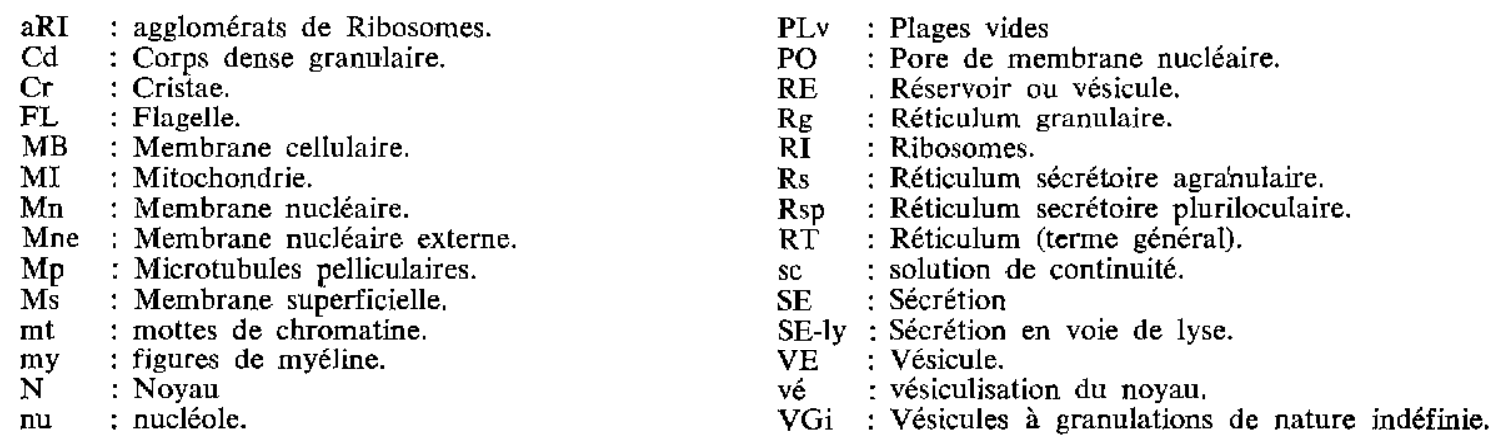


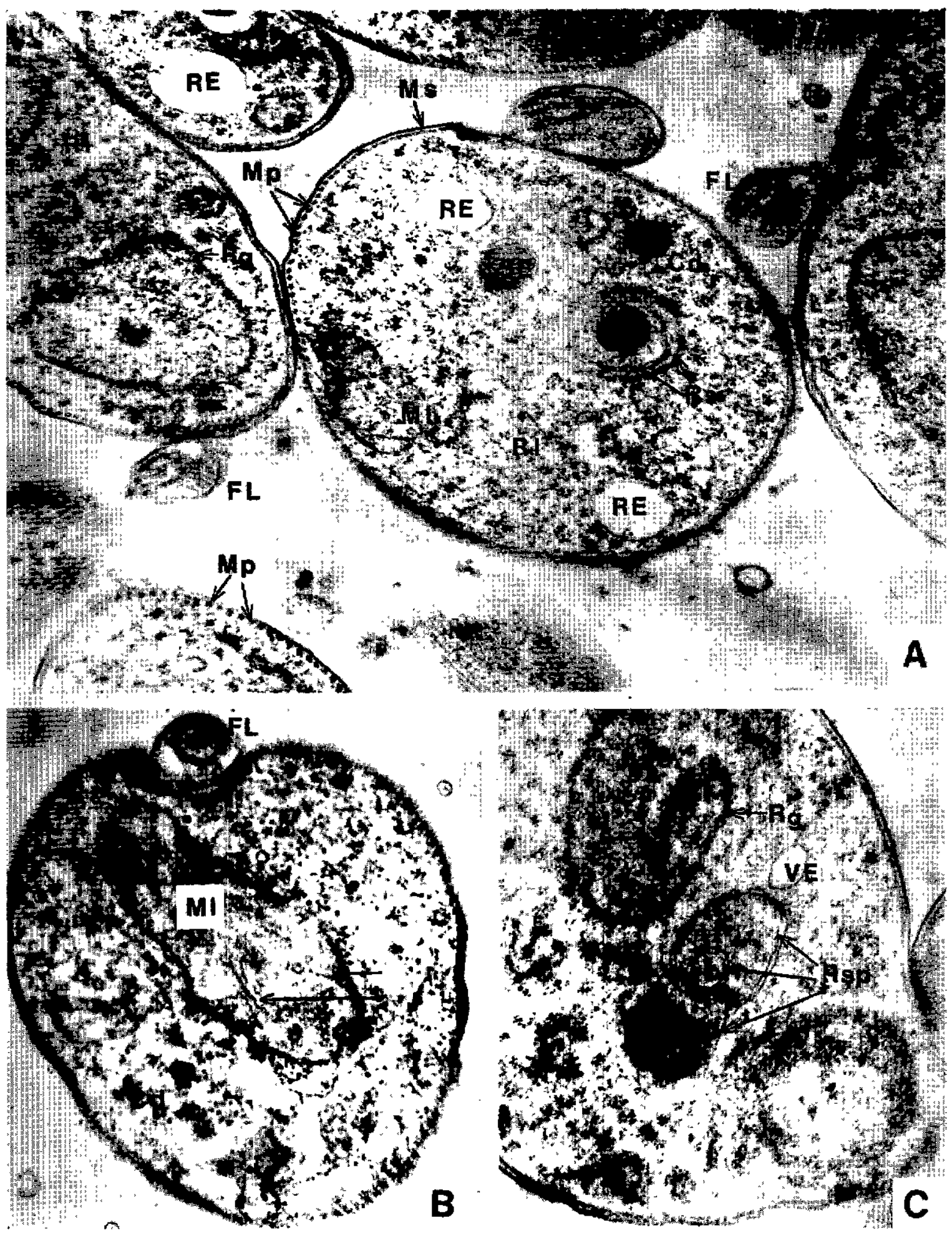

Planche I. - T, vpanosoma congolense: morphologie normale. Grossissement A : $21.300 ;$ B : $26.400 ; \mathrm{C}: 26.400$. 

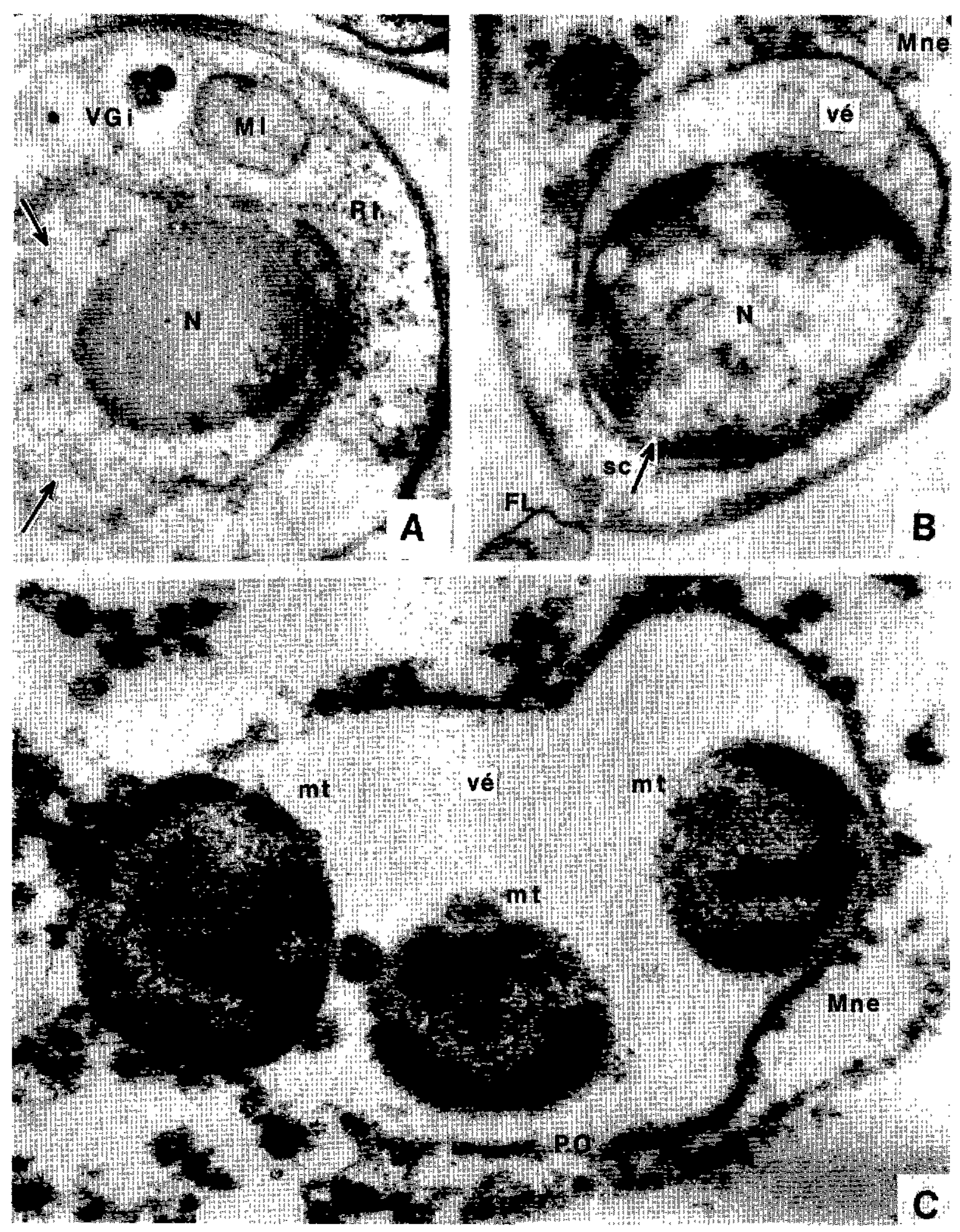

Planche II. - A. - Trypanosona congolense + Bérénil $100 \mu \mathrm{g} / \mathrm{ml}$ pendant 16 heures, G: 37.200;

B. $-T$. congolense + Prothidium $50 \mu \mathrm{g} / \mathrm{ml}$ pendant 15 heures, $\mathrm{G}: 31.800 ; \mathrm{C} .-T$. brucei + Bérénil $100 \mu \mathrm{g} / \mathrm{m}]$ pendant 18 heures, $G$ : 40500 . 


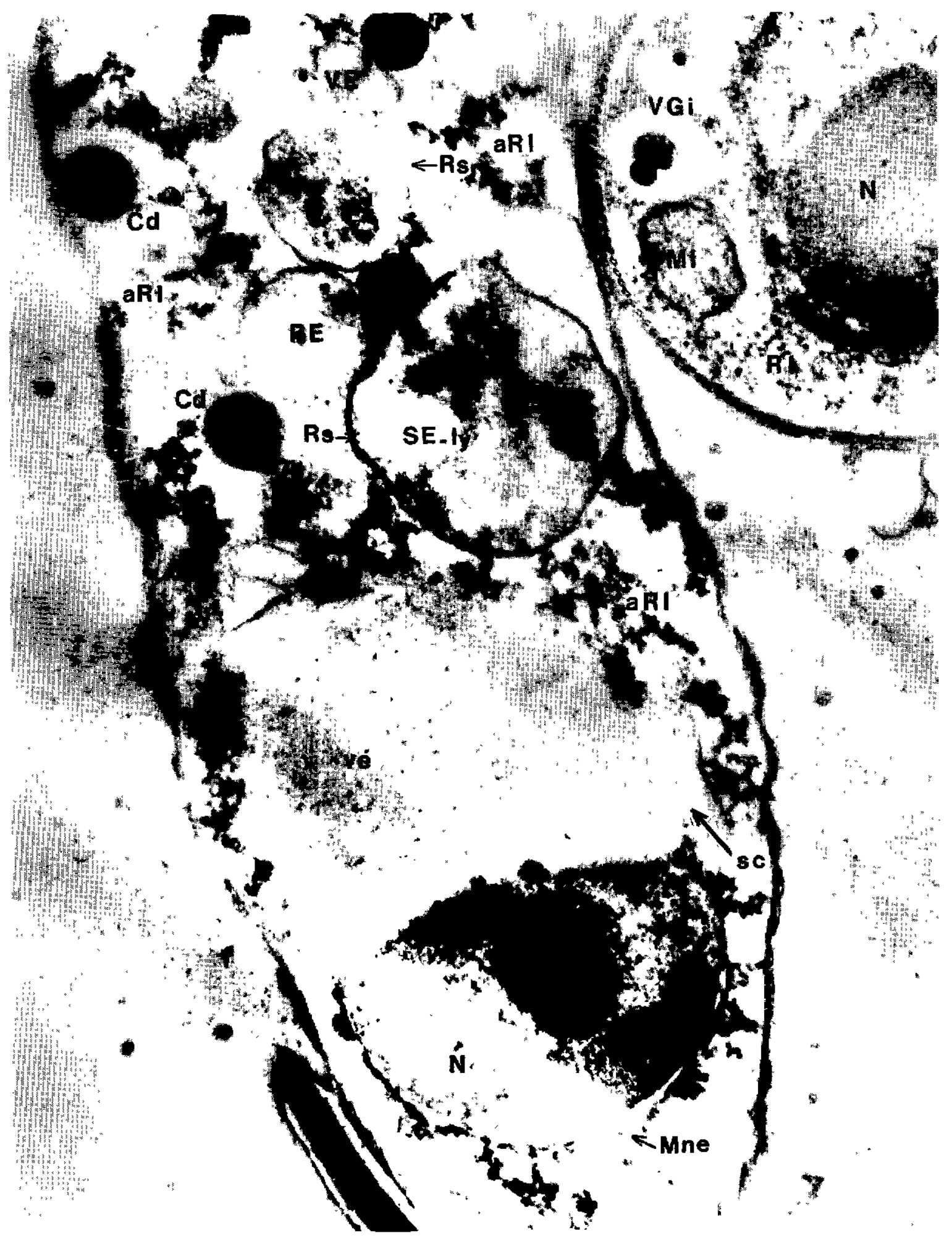

Planche III. - Trypanosoma congolense + Bérénıl $100 \mathrm{ug}, \mathrm{ml}$ pendant 16 heures, G: 37.200 . 


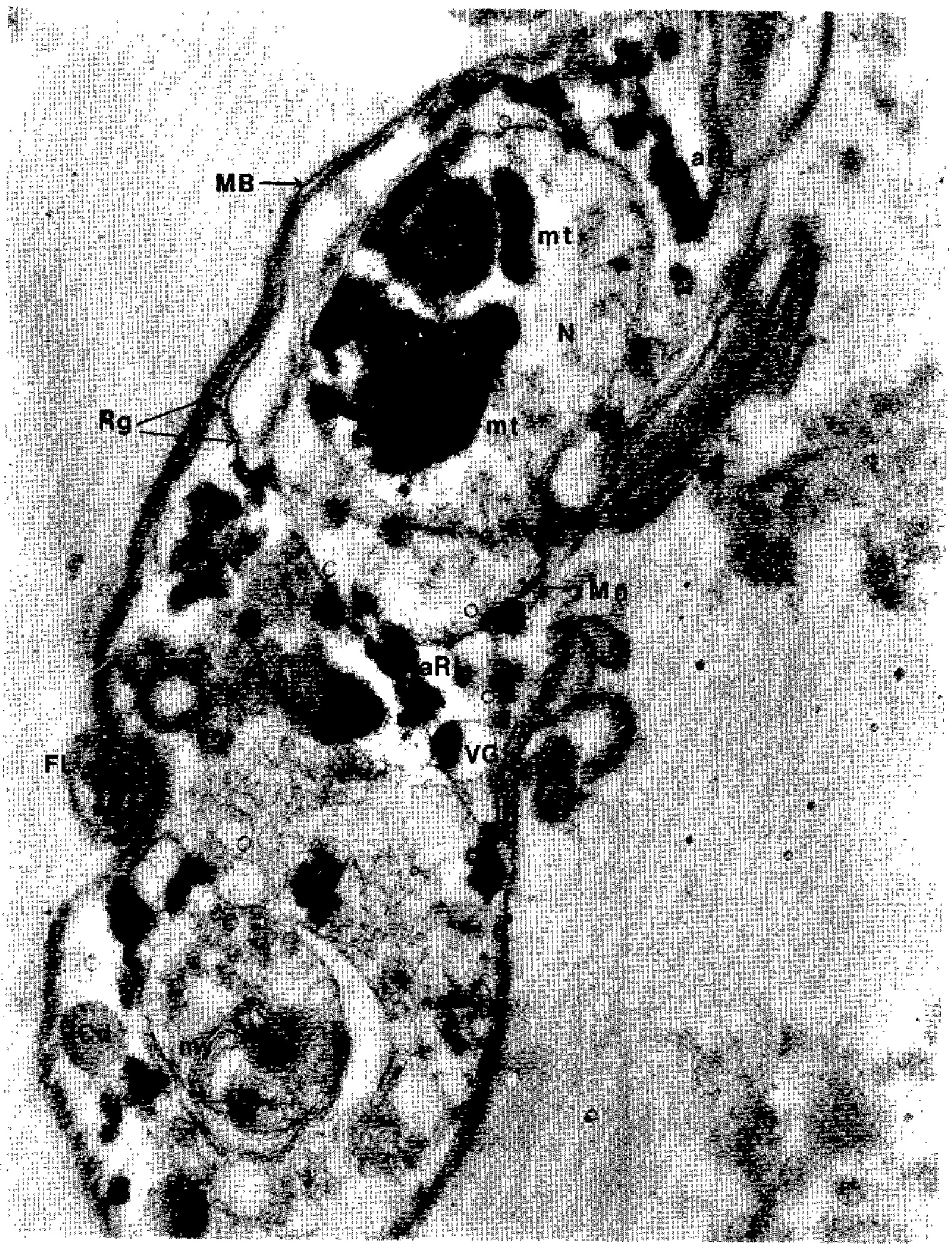

Planche IV. - Trypanosoma congolense + Ethidium $25 \mu \mathrm{g} / \mathrm{ml}$ pendant 18 heures, G : 26.400. 


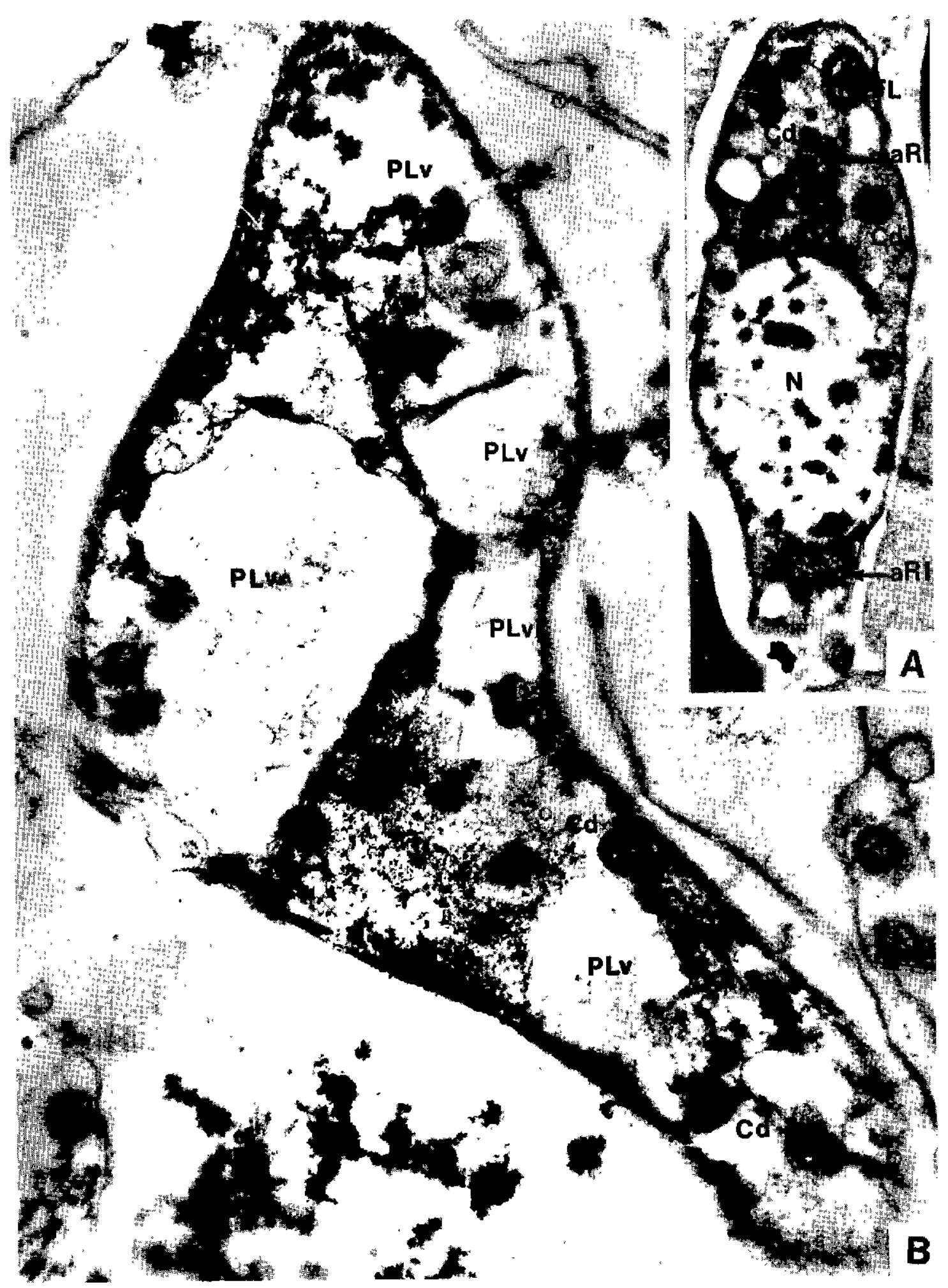

Planche V. - A. - Trypanosoma virax + Antrycide $200 \mu \mathrm{g} / \mathrm{ml}$ pendant 18 heures, G: 11.300; B. - T. brucei + Antrycide $200 \mu \mathrm{g} / \mathrm{ml}$ pendant 48 heures, G : 23.000. 

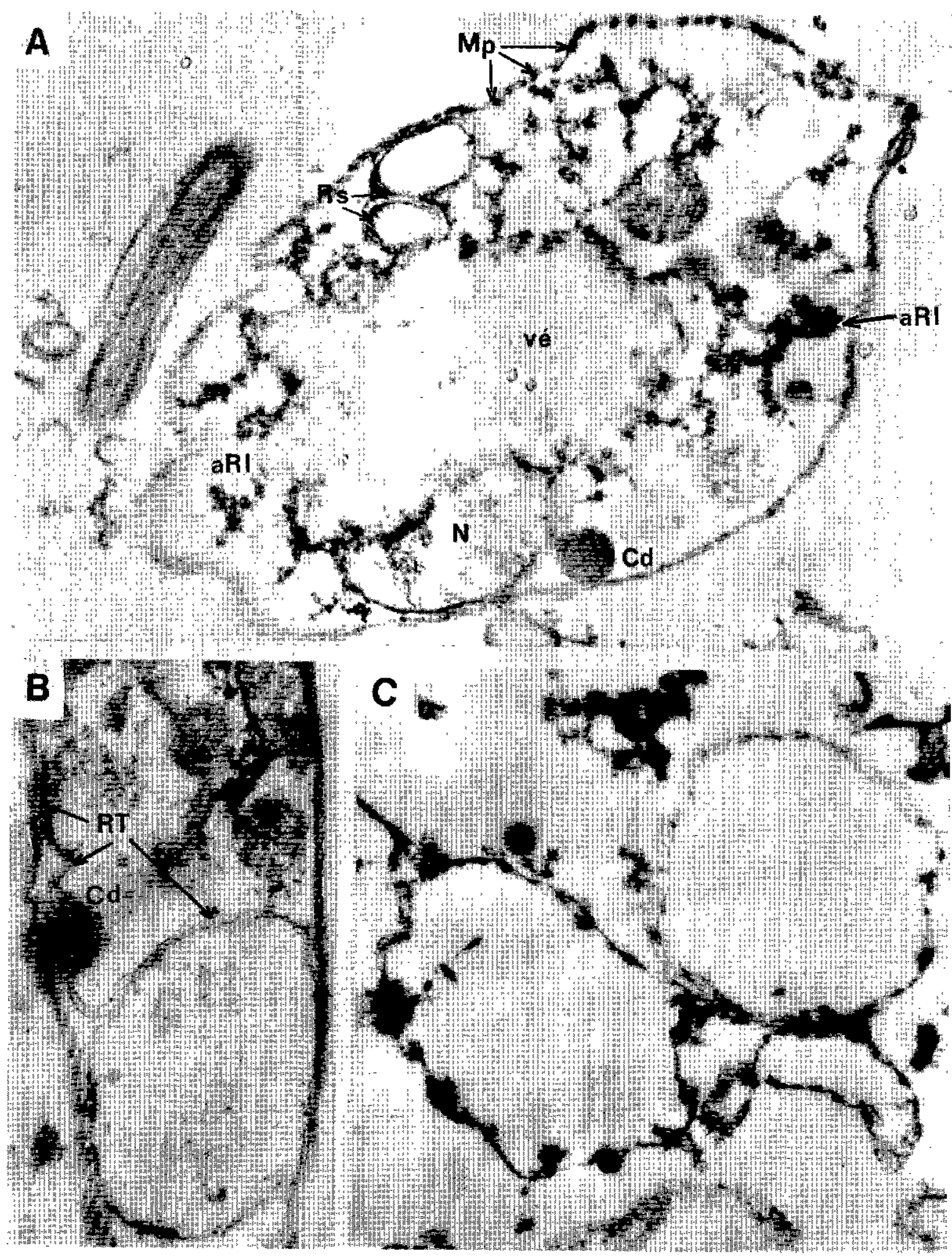

Planche Vl. - A. - Trypanosoma congolense + Bérénil $100 \mu \mathrm{g} / \mathrm{ml}$ pendant 18 heures, G: 26.400;

B. - T. congolense + Bérénil $100 \mu \mathrm{g} / \mathrm{ml}$ pendant 18 heures, G:37.200; C. - T. brucei + Béténil $100 \mu \mathrm{g} / \mathrm{ml}$ pendant 18 heures, $\mathrm{G}: 26.400$. 


\section{SUMMARY}

\section{Ultrastructural study of effects produced on trypanosomes by some trypanocidal drugs}

The main 1dea which directed this paper was the actual importance of such studies as microphysiology and ultrastructural morphology in trypanosomiasis research. The following are the results from electronic microscope observation of trypanosomes which were incubated in vitro in trypanocidal drug solution during 15 to 18 hours. Three trypanosome species were used in this experiment: Trypanosoma congolense. $T$ brucei, $T$. vivax and the drugs were $=$ Quinapyramine methylsulfate at concentration of $200 \mu \mathrm{g} / \mathrm{ml}$, Dinamizene $(100 \mu \mathrm{g} / \mathrm{ml})$, Prothidium $(50 \mu \mathrm{g} / \mathrm{ml})$, Ethidium $(25 \mu \mathrm{g} / \mathrm{ml})$.

The injuries in the body of the trypanosomes appeared as nuclear vesiculsation and disruption of the nucleus. The kinetoplast and the mitochondrial structures were early removed. The flagella remained longer and so did the endoplasmic reticulum and the secretory agranular reticulum. The ribosomes agglomerated and led to formation of empty areas into the cell. The cytoplasmic membrane and the pellicular microtubules were not much damaged

These observations are discussed and compared to results from some other species of trypanosomes. Six plates joined to the paper.

\section{RESUMEN}

\section{Estudio ultra-estructural de los efectos producidos por varios medicamentos tripanocidos sobre tripanosomos}

Se presentan los resultados de observaciones hechas con el microscopio electrónico sobre tripanosomos tratados por medicamentos in vitro durante 15 a 18 horas. Se experımentaron tres especies de tripanosomos. Trypanosoma congolense. $T$. brucei, y $T$. vivax. Los medicamentos utilizados son: el metilsulfato de Quinapiramina a razón de $200 \mu \mathrm{g} / \mathrm{ml}$, el Dinamizeno $(100 \mu \mathrm{g} / \mathrm{ml})$, el Prothıdium $(50 \mu \mathrm{g} / \mathrm{ml})$, el Ethidium $(25 \mu \mathrm{g} / \mathrm{ml})$, el Isometamidium $(25 \mu / \mathrm{ml})$.

Provocan en los tripanosomos la vesiculación del núcleo y su fragmentación en terrones. El kinetoplasmo y las estructuras de las mitocondrias desaparecen precozmente. El flagelo persiste mucho tiempo ası como el retículo endoplasmico y el retículo secretorio. Se aglomeran los ribosomos y dejan espacios vacios. La membrana citoplasmica y las microtubulas son poco modificadas.

Se discuten y se comparan dichas observaciones - ilustradas por 6 series de fotografias - con trabajos sobre algunas otras especies de tripanosomos.

\section{BIBLIOGRAPHIE}

1. BIRD (R. G.), MOLLOY (J.O.) et ORMEROD (W. E.), «Granules and tubules in the cytoplasm of the sleeping sickness trypanosome. an electron microscope study ", Trans. Roy. Soc. Irop. Med. Hyg., 1966, 60 : 753-60.

2. BOISSON (M. E.), BOISSON (C.) et MATTEI (X.), «Présence d'un appareil élaborateur singulier chez Trypanosoma congolense», $C . R$ Soc. Biol., 1967, 161 (8-9) : 1772-74.

3. GUTTERIDGE (W. E.), "Some effects of Pentamidine di-isethionate on Crithidia fasciculata». J. Protozool, 1969, 16 (2): 306-11.

4. HERMAN (R.), «Acriflavin - induced dyskinetoplastic Leishmania donovani grown in monkey kidney cell culture », J. Protozool., 1968, 15 (1): 35-44.

5. HILL (G.C.), BROWN (C. A.) et CLARK (M. V.), "Structure and function of Mitochondria in Crithidia fasciculata », J. Protozool., 1968. 15 : 102-09.
6. JADIN (J.M.) et CREEMERS (J.), « L'ultrastructure des formes en rosace de Leishmania tropica Wright, 1903 ", Ann. Soc. Belge Méd. trop., 1966, 46 (3) : 349-54.

7. KILLICK - KENDRICK (R.), « The apparent loss of the kunetoplast of Tiypanosoma evansi after treatment of an experimentally infected horse with Berenil ", Ann, trop. Méd Parasitol, $1964,58,481-501$.

8. LANTZ (M.), KAHAN (D.) et ZAHALSKY (A. C.), «Interaction of trypanocidal agents with soluble RNA 》, Abstract $n^{\circ} 76$ from the 21st annual Meeting of the Society of Protozoologists, Columbus, Ohio, 1968, Sept. 3-7, in : J. Protozool., 1968, 15, supp].

9. MACADAM (R. F.) el WILLIAMSON (J.), «The effect of drugs on the fine structure of trypanosomes *, Parasitology, 1967, 57, 21 P (proceedings). 
10. MACADAM (R. F.) et WILLIAMSON (J.), "Lesions in the fine structure of Trypanosoma thodestense specifically associated with drug treatment ", Trans. R. Soc. trop. Med. Hyg. 1969 , 63 (4) : 421-22.

11. ORMEROD (W. E.), « The appearance of "volutin granules" in Trypanosoma rhodesiense studied by phase - contrast and electron microscopy », Parasitology, 1962, 52 (3-4): 9 P - 10 P (proceedings).

12. RIOU (G.), «Disparition de l'A.D.N. du kinetoplaste de Trypanosoma cruzi cultivé en présence de Bromure d'Ethidium ", C. R. Acad. Sci., Paris, 1968, 266 (3) : 250-52.

13. TOURE (S. M.), "Cinétique de la mort in vitro de Trypanosoma vivax et $T$. congolense au contact de médicaments trypanocides », Rev. Elev. Méd. vét. Pays trop., 1967, 20 (1): 109-14.

14. VICKERMAN (K.), a Polymorphism and mitochondrial activity in sleeping sickness trypanosomes ", Nature, London, 1965, 208: 762-66.
15. VICKERMAN (K.), * Electron microscopy of tsetse salivary gland stages in the life cycle of Trypanosoma rhodesiense», Trans. Roy. Soc. trop. Med. Hyg., 1966, 60:8.

16. VICKERMAN (K.), «The fine structure of Trypanosoma congolense in its bloodstream phase *, J. Protozool., 1968, 16 (1): 54-69.

17. VICKERMAN (K.) et LUCKINS (A, G.), « Localization of variable antigens in the surface coat of Trypanosoma brucei using ferritin conjugated antibody », Nature, London, 1969, 224, 11.25-26.

18. WILLIAMSON (J.), «The activity of drugs on Trypanosoma congolense in vitro at $37^{\circ} \mathrm{C} »$, Trans. Roy. Soc. trop. Med. Hyg., 1969, 63 (4): 422-23.

19. WILLIAMSON (J.) et MACADAM (R. F.), "Effects of trypanocidal drugs on the fine structure of Trypanosoma rhodesiense *, Trans. Roy. Soc. trop. Med. Hyg. 1965, 59: 367-68. 